\title{
Near-Infrared Spectroscopy In atypical Depression: A Diagnostic Possibility
}

\author{
Takeaki Takeuchi*, Kazuaki Hashimoto and Masahiro Hashizume \\ Department of Psychosomatic Medicine, Toho University School of Medicine, Japan \\ *Corresponding author: Takeaki Takeuchi, Department of Psychosomatic Medicine, Toho University School of \\ Medicine, Tokyo, Japan
}

\section{ARTICLE INFO}

Received: 豐 June 29, 2019

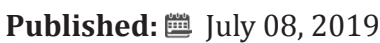

Citation: Takeaki Takeuchi, Kazuaki Hashimoto and Masahiro Hashizume. Near-Infrared Spectroscopy In atypical Depression: A Diagnostic Possibility. Biomed J Sci \& Tech Res 19(3)-2019. BJSTR. MS.ID.003303.

\begin{abstract}
Optical topography is a non-invasive device with high resolution for imaging hemodynamic changes to assess brain function, using near-infrared spectroscopy (NIRS). In Japan, the cost of this technique can be covered by healthcare insurance for differentiating depressive symptoms. Although atypical depression is often seen in psychosomatic clinics and known to have a relationship with other physiological disorders, such as metabolic syndrome and other physical conditions, NIRS findings are limited to understanding the neurophysiological aspects of atypical depression. In this brief paper, we address the possible role of NIRS diagnostic accuracy for depression and show cases of NIRS diagnosis in atypical depression that relate to the body and mind, in contrast to melancholic types of depression and bipolar disorders. This article could help psychosomatic medicine practitioners when debating the similarities and differences between depressions in psychosomatic illness.
\end{abstract}

Keywords: Atypical depression; Bipolar disorder; Near-infrared spectroscopy; Melancholic depression; Optical topography

\section{Introduction}

Near-infrared spectroscopy (NIRS) is a non-invasive technique using relatively small and portable equipment, with high temporal resolution for imaging hemodynamic changes to assess brain function [1-4]. This device was first introduced by Jöbsis in 1977, which allowed measurement of tissue components [5]. NIRS can detect brain function by evaluating changes in the concentration of oxygenated hemoglobin, deoxygenated hemoglobin, totalhemoglobin or cytochrome-c-oxidase [6] using near-infrared light. In addition, it has been used to resolve changes in the combination of hemoglobin and cytochrome-c-oxidase, which has led to many clinical applications for measurements in the human brain [6]. NIRS has a high resolution, it can be used to detect a similar cortical activation pattern to functional magnetic resonance imaging (fMRI) [7], positron emission tomography (PET) [8], and single photon emission computed tomography (SPECT) [9]. NIRS was largely unknown to clinicians in mental health in Japan; however, since 2009 it has been approved by the Ministry of Health, Labor and Welfare as an advanced medical technology to differentiate psychiatric diagnosis [10].
The cost of this technique has been covered by healthcare insurance to differentiate major depressive disorder and bipolar disorder since 2014. Therefore, many Japanese psychiatric hospitals and university affiliated hospitals have introduced NIRS for diagnostic accuracy in depression both for clinical and research purposes. Major depressive disorder was ranked as the third leading contributor to the global burden of disease in 2004 and is expected to move to first place by 2030 [11]. Depression is associated with physiological disorders, such as metabolic syndrome and other physical conditions. Of the various specifiers created for major depressive disorder to help clinicians for its treatment, atypical depression is thought to have the strongest relationship to lifestyle diseases [12,13]. More specifically, according to the Diagnostic and Statistical Manual of Mental Disorders, Fifth Edition (DSM5), atypical depression is defined as a condition presenting with mood reactivity plus at least two of the following four symptoms: hyperphagia, hypersomnia, laden paralysis, and interpersonal rejection sensitivity [14]. 
Of these, hyperphagia and hypersomnia are directly related to lifestyle. Psychosomatic physicians treat mental disorder and somatic symptoms; therefore, NIRS finding for atypical depression will assist with their diagnosis and treatment. Further, psychiatric diagnosis is based on the subjective report of symptoms and observed behavior. Therefore, NIRS, as a technique for the diagnosis of depression, can facilitate the clinical process. Recently, we reported increases in the diagnostic accuracy for depression using the Beck depression inventory II (self-report inventory) with NIRS (which was written in Japanese, no reference). These findings may be helpful for both internists and psychiatrists. Furthermore, differential diagnosis for subtypes of depressive disorders (such as melancholic and atypical) and bipolar disorder is difficult for mental professionals [15]; therefore, treatment decisions for each disorder are different.

In addition, some studies have addressed the overlap between atypical depression and bipolar disorder, especially type II, with conflicting findings [16-18]. Atypical depression can meet the criteria for bipolar disorder type II $[16,18]$; therefore, the difference between atypical depression and bipolar disorder is vague and, to the best of our knowledge, few studies have assessed NIRS images in atypical depression. Therefore, recognizing the difference between melancholic depression, atypical depression, and bipolar disorder would be useful in clinical medicine. In this report, we addressed the possible role of NIRS in the diagnostic accuracy of depression. In addition, we describe three cases of NIRS diagnosis in atypical depression that are related to body and mind symptoms, in contrast to melancholic types of depression and bipolar disorders.

\section{Methods}

\section{Participants and Diagnosis of Depression}

We extracted the data of 57 participants who were diagnosed with major depressive disorder or bipolar disorder, who were admitted to Toho University Hospital from June 2015 to June 2017. Patients were diagnosed by a clinician (specializing in psychosomatic psychiatry) and evaluated using the Beck Depression Inventory (BDI)-II and NIRS. Sixteen people were excluded because of a lack of data. Therefore, we used data from 41 people for this study (mean age $=43.4$ years old, SD $=14.1$ years, 24 males and 17 females). The diagnoses of all extracted data were rechecked by two physicians who specialize in psychiatry and psychosomatic medicine according to DSM-5 criteria [14]. Assessors were blinded to the NIRS data at the point of diagnosis.

\section{Statistical Analyses and Ethical Consideration}

After the characteristics of the study population were descriptively evaluated according to the BDI-II low and high scores, we calculated the cut off value for depression using the Yoden index of the depression questionnaire, based on our previous study [19] We compared the accuracy of the depression diagnosis using the centroid value. We evaluated diagnostic accuracy using area under the curve (AUC) analysis. All analyses were performed using the EZR
Ver 1.32 [20] statistical package. Data are expressed as means and SD. $\mathrm{P}<0.05$ (two-tailed), were considered statistically significant. Two-tailed t-tests were used to compare continuous values, and Fisher's exact or proportion tests were used to compare categorical values between the two groups.

We extracted three clinical cases from these data for case presentation. All three clinical cases underwent a structured clinical interview using questions from the major depressive disorder and bipolar disorder section of the of the Mini International Neuropsychiatric Interview [21]. Two physicians who specialize in both psychiatry and psychosomatic medicine diagnosed melancholic depression, atypical depression, or bipolar disorder according to DSM-5 criteria [14]. The two physicians who diagnosed the cases were blinded to the NIRS data at the point of diagnosis. This clinical study was carried out in accordance with the latest version of the Declaration of Helsinki and was approved by the ethics committee of the Toho University Faculty of Medicine (registration number M17141 and M17246). Informed consent was obtained from all patients, following an explanation of the NIRS procedure.

\section{NIRS Device in the Study and Depression Diagnosis Assisted by NIRS}

A fifty-two channel NIRS (ETG-one 4000; Hitachi Medical, Tokyo, Japan) was used to derive values for oxygenated hemoglobin and deoxygenated hemoglobin using two wavelengths (695 and 830 $\mathrm{nm})$. We placed 18 channels on the frontal cortex and 36 channels over the right and left temporal cortex. We used the device settings reported in previous studies [22,23]. Participants sat comfortably in a quiet room. The task procedures were explained by a clinical technician, who subsequently monitored head movement during the procedure. During the verbal fluency task (VFT) [24], participants were instructed to look at a device monitor from external speakers. The VFT was conducted according to previous studies [22,25]. Briefly, the procedure consisted of a pre-task (30 seconds), VFT (60 seconds), and post-task (70 seconds) stage. During the preand post-task periods, participants were instructed to speak five Japanese vowels $(\mathrm{a}, \mathrm{i}, \mathrm{u}, \mathrm{e}, \mathrm{o})$. The subtraction method (task minus pre- and post-task) was used to minimize any vocalization effects during the VFT. Participants were instructed to speak as many Japanese words as possible from the beginning with a designated syllable [22].

To avoid artifact effects, noise, and head movement data (bad channels) were deleted by three clinicians who are independent to the study. Based on a previous study $[25,26]$, the evaluation of depression was performed by two simple visual indices, such as "integral value" and "centroid value". The integral value describes the size of the hemodynamic response, whereas the centroid value serves as an index of time-course changes throughout the task [26]. The threshold value to differentiate major depressive disorder to non-major depressive disorder was $54 \mathrm{~s}$ lower than the centroid value in frontal lobe. Using this threshold, $74.6 \%$ of the 
patients with major depressive disorder were classified correctly [26]. The integral value represents patients with depression who had significantly lower prefrontal activation when compared with healthy people [27-29]. This was calculated by differences in clinical symptoms (such as the Global Assessment of Functioning, GAF, score) between major depressive disorder and bipolar disorder with depressive symptoms.

\section{Results}

\section{NIRS Diagnostic Accuracy with Subjective Depressed Mood Severity}

The mean Beck Depression Inventory (BDI)-II score, NIRS centroid value and NIRS integral value in the 41 participants were
29.8 (standard deviations, S.D., 12.6), 52.8 (S.D. 11.3), and 67.3 (S.D. $62.2)$, respectively. The subjects were classified into high $(n=24)$ and low $(n=17)$ scores at 26 points of BDI-II. The demographics of the low and high groups for BDI-II are shown in Table 1. The diagnostic accuracy between BDI-II alone and BDI-II with NIRS is shown in Table 2. The specificity, AUC, and positive likelihood ratio of NIRS depression diagnosis are higher than the BDI-II depression diagnosis. In contrast, sensitivity was higher in the BDI-II diagnosis. When comparing the BDI-II low and high scores with NIRS, sensitivity, specificity, AUC, and positive likelihood ratios were higher in the low score group when compared with the higher group. The demographics between depressive disordes and biplar disorder II are shown in Table 3.

Table 1: Characteristics between BDI-II low and high group $(\mathrm{n}=41)$.

\begin{tabular}{|c|c|c|c|}
\hline & low score(n = 17) & high score(n = 24) & p value \\
\hline Gender & & & 0.21 \\
\hline Male & 12 & 12 & \\
\hline Female & 5 & $44.8 \pm 15.2$ & 0.78 \\
\hline Age & $42.7 \pm 12.8$ & $17.5 \pm 29.3$ & 0.07 \\
\hline Disease duration (months) & $41.2 \pm 52.5$ & 15 & 0.08 \\
\hline Depressive Disorders & 15 & 9 & \\
\hline Bipolar and Related Disorders & 2 & & \\
\hline
\end{tabular}

BDI-II. Beck depression inventory II. Two-tailed t-tests were used to compare the mean differences. For categorical variables, Fisher's exact tests or proportion tests were used.

Table 2: Comparison of diagnostic accuracy between BDI-II and BDI-II with NIRS.

\begin{tabular}{|c|c|c|c|c|c|c|}
\hline & $\begin{array}{c}\text { Cutoff value } \\
\text { (Youden index) }\end{array}$ & Sensitivity & Specificity & $\begin{array}{c}\text { Area under the } \\
\text { Roc curve }\end{array}$ & $\begin{array}{c}\text { Positive } \\
\text { likelihood ratio }\end{array}$ & $\begin{array}{c}\text { Negative } \\
\text { likelihood ratio }\end{array}$ \\
\hline BDI-II (n= 41) & 26 & 81.8 & 50 & 0.59 & 1.63 & 0.61 \\
\hline $\begin{array}{c}\text { NIRS centroid } \\
\text { value (n= 41) }\end{array}$ & 55.1 & 72.7 & 70 & 0.69 & 2.42 & 0.41 \\
\hline $\begin{array}{c}\text { NIRS centroid } \\
\text { values in BDI-II } \\
\text { low score (n = 17) }\end{array}$ & 55.1 & 100 & 73.3 & 0.83 & 3.74 & 0 \\
\hline $\begin{array}{c}\text { NIRS centroid } \\
\text { values in BDI-II } \\
\text { high score (n=24) }\end{array}$ & 53.8 & 66.7 & 66.7 & 0.63 & 2 & 0.5 \\
\hline
\end{tabular}

Table 3: Characteristics between depression and bipolar disorder $(\mathrm{n}=41)$.

\begin{tabular}{|c|c|c|c|}
\hline & Major depressive disorders $(\mathbf{n}=\mathbf{3 0})$ & bipolar disorder II (n = 11) & p value \\
\hline Gender & & & 0.47 \\
\hline Male & 19 & 5 & \\
\hline Female & 11 & 6 & 0.2 \\
\hline Age & $45.1 \pm 14.0$ & $38.7 \pm 14.0$ & 0.09 \\
\hline Disease duration(month) & $33.9 \pm 45.9$ & $9.5 \pm 18.6$ & 0.44 \\
\hline BDI-II & $28.9 \pm 12.6$ & $32.3 \pm 12.8$ & \\
\hline
\end{tabular}

BDI-II. Beck depression inventory II. Three-tailed t-tests were used to compare the mean differences. For categorical variables, Fisher's exact tests or proportion tests were used. Participants with major depression includes melancholic depression and atypical depression used for case presentation.

\section{Case 1: NIRS Image for Melancholic Depression}

A 54-year-old male presented at our out-patient service with fatigue as a main symptom. The first onset of fatigue symptoms appeared before he went to work when he was 48 years old. His symptoms deteriorated and fluctuated depending on his workload. He could tackle his symptoms by himself; however, combined with 
the depressive mood, he sought help from the local doctor and was referred to our university hospital. He presented with sleep apnea syndrome as a coexisting illness. He reported no family history, except paternal acute myocardial infarction. Delusion and hallucinations were not observed. He had been prescribed 5 mg olanzapine (chlorpromazine equivalent dose, $200 \mathrm{mg}$ ). The GAF score was 55. Other diseases that because depressive moods were ruled out by blood test including thyroidal, adrenal and iron function. He was diagnosed with major depressive disorder, moderate, melancholic type, according to the DSM-5. The mean centroid value was $50.4 \mathrm{~s}$ in the frontal lobes; therefore, this was classified as major depressive disorder. The mean integral value of 57.8 indicated that the clinical symptom of depression (GAF) was mild to moderate (Figure 1).

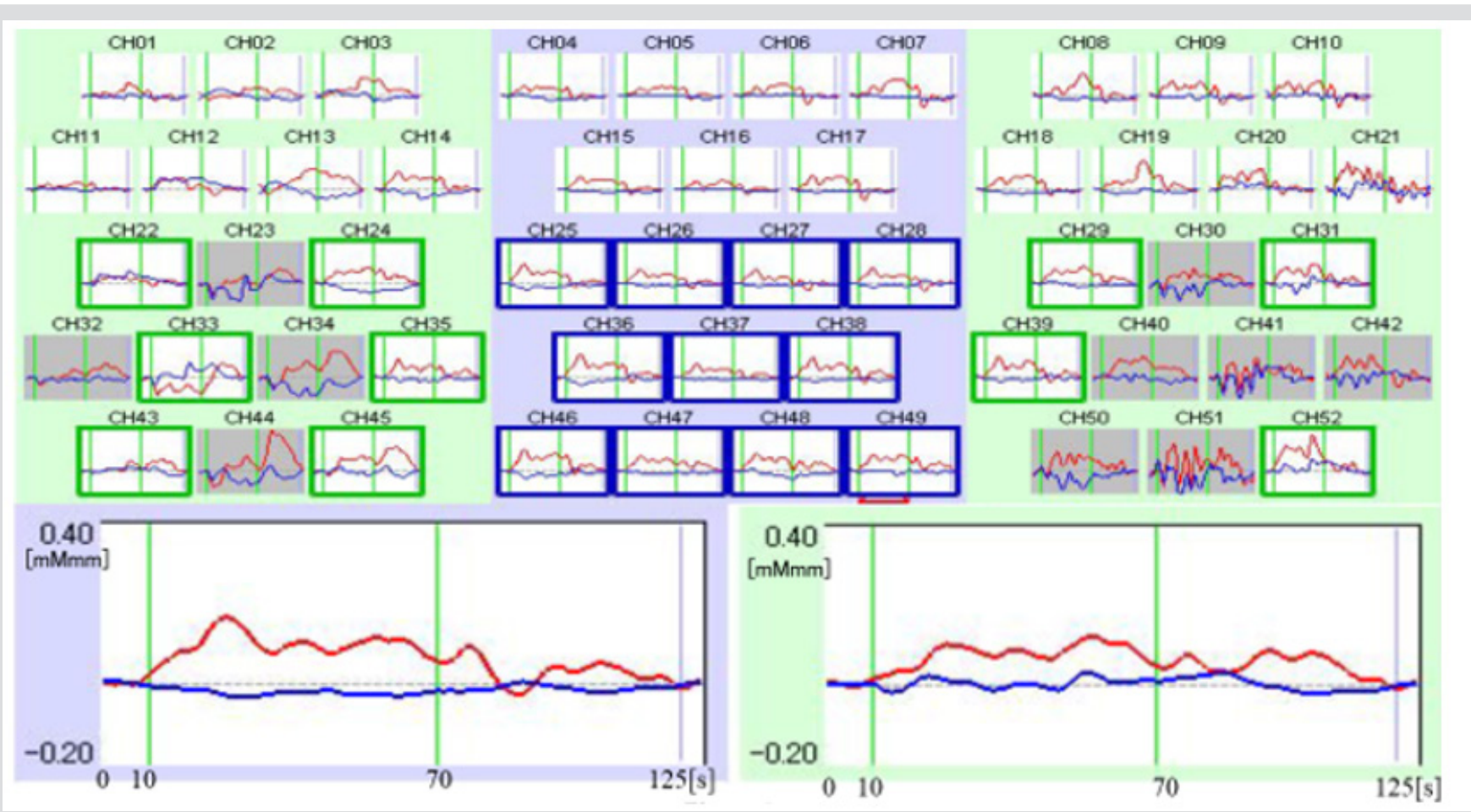

Figure 1: For melancholic depression. Upper part represents 52 channel row data of melancholic depression (51 years old man), upper left: right temporal lobes, center: frontal lobes, upper right: left temporal lobes. Lower left: mean value of $11 \mathrm{frontal}$ lobes data (Integral value 57.8, Centroid value 50.4s). Lower right: mean value of selected 10 temporal lobes data without artifact (Integral value 46.0, Centroid value 60.1s). Integral analysis used the last $10 \mathrm{~s}$ of the pre-task, $60 \mathrm{~s}$ task, and first $55 \mathrm{~s}$ of the posttask $=125 \mathrm{~s}$ in total. Red line: oxyhemoglobin concentration, Blue line: deoxyhemoglobin concentration.

\section{Case 2: NIRS Image for Atypical Depression}

A 24-year-old female presented at our out-patient service with irritability as a main symptom. The first onset of irritability appeared when she conducted her junior schoolwork as a teacher when she was 22 years old. With the help of a school counselor, she took rest and reduced her workload; however, her fatigue symptoms remained. She did not have a coexisting illness, past medical history or any family history of specific diseases. Delusions or hallucinations were not observed. She had been prescribed $20 \mathrm{mg}$ escitalopram oxalate, 20 lithium carbonate, and $6 \mathrm{mg}$ bromazepam. Her GAF score was 65. Other diseases that cause a depressive mood were ruled out by blood test including thyroidal, adrenal and iron function. She was diagnosed with major depressive disorder, moderate, atypical type according to the DSM-5. Her mean centroid value was $37.7 \mathrm{~s}$ in the frontal lobes, which was classified as major depressive disorder. The mean integral value of 5.6 indicated that the clinical symptom of depression was moderate to severe (Figure 2). 


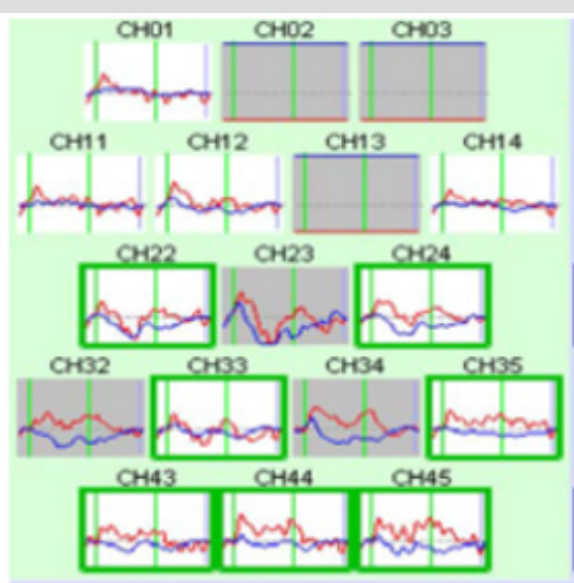

0.40 $[\mathrm{mMmm}]$

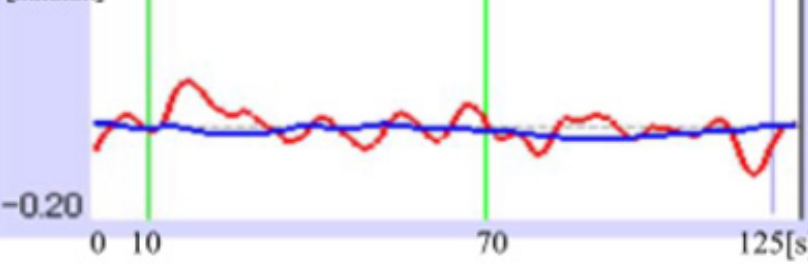

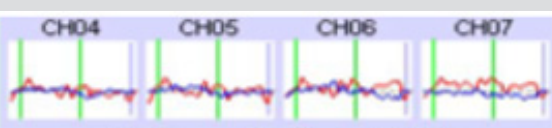
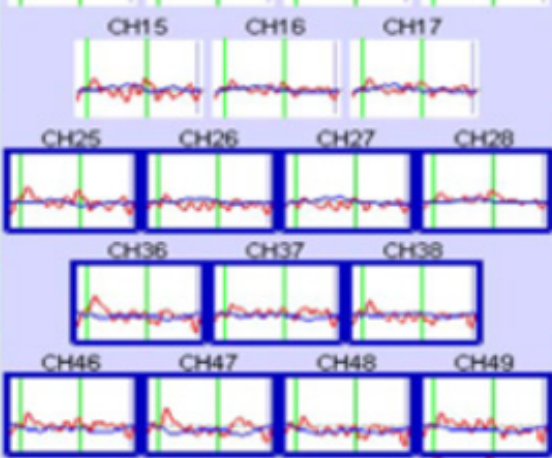

0.40

$[\mathrm{mMmm}]$

$0 \quad 10$
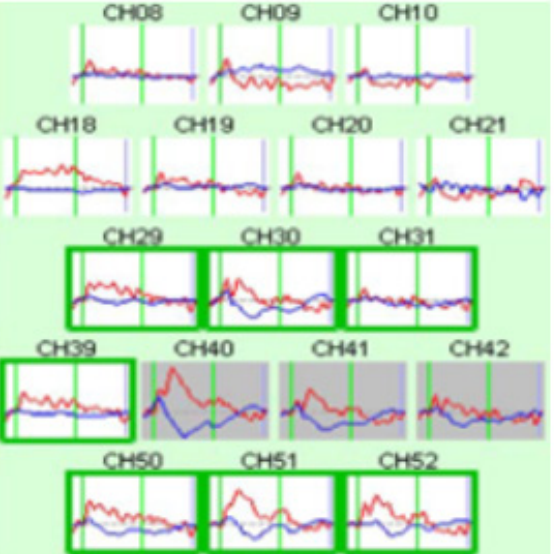

it

Figure 2: For atypical depression. Upper part represents 52 channel row data of atypical depression ( 25 years old woman), upper left: right temporal lobes, center: frontal lobes, upper right: left temporal lobes. Lower left: mean value of 11 frontal lobes data (Integral value 5.6, Centroid value 37.7s). Lower right: mean value of selected 14 temporal lobes data without artifact (Integral value 30.4, Centroid value 41.3s). Integral analysis used the last $10 \mathrm{~s}$ of the pre-task, $60 \mathrm{~s}$ task, and first $55 \mathrm{~s}$ of the posttask $=125 \mathrm{~s}$ in total. Red line: oxyhemoglobin concentration, Blue line: deoxyhemoglobin concentration.

\section{Case 3: NIRS Image for Bipolar Disorder II}

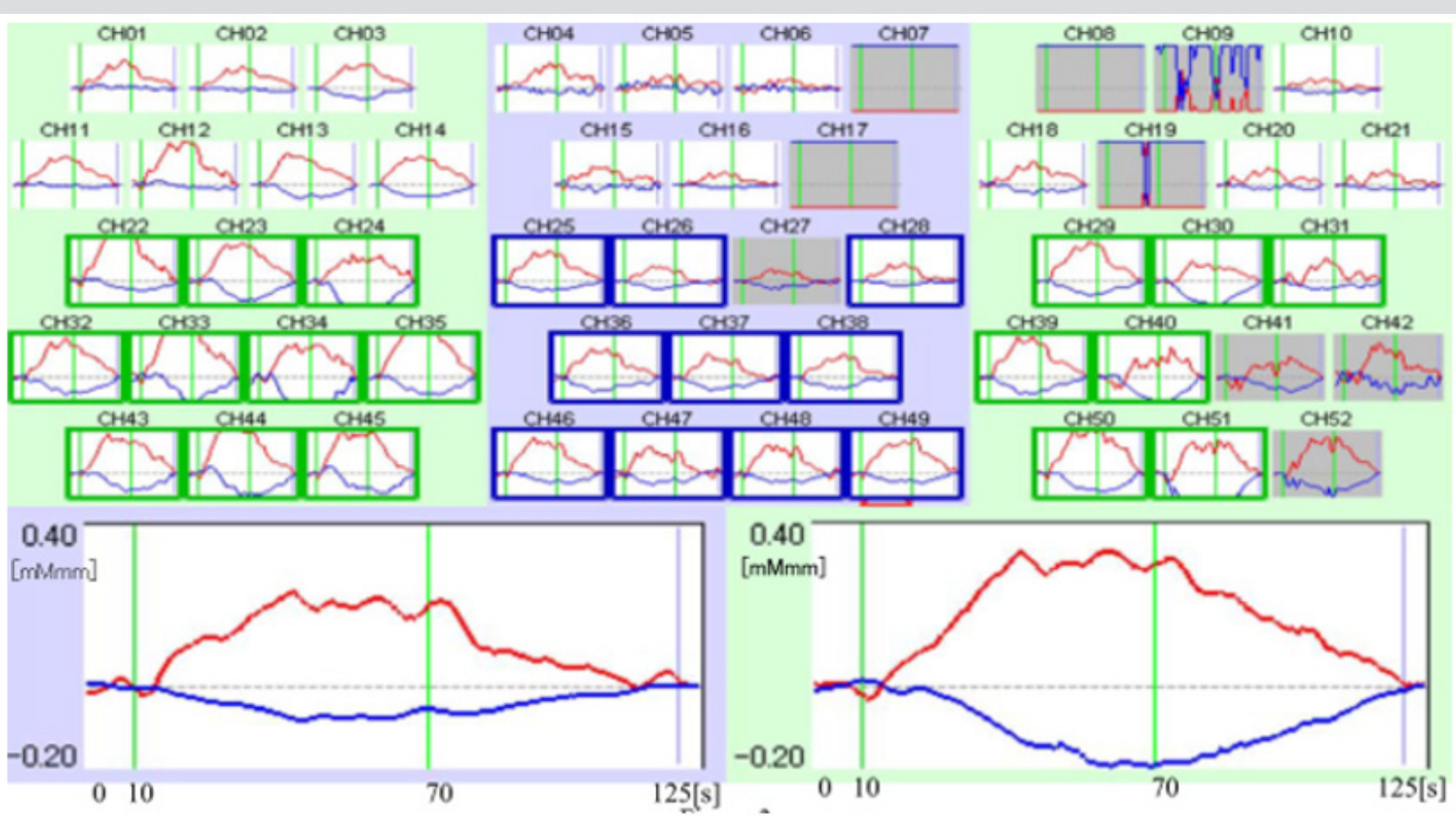

Figure 3: For bipolar disorder II. Upper part represents 52 channel row data of bipolar disorder type II (26 years old woman), upper left: right temporal lobes, center: frontal lobes, upper right: left temporal lobes. Lower left: mean value of 10 selected frontal lobes data without artifact (Integral value 93.2, Centroid value 56.2s). Lower right: mean value of selected 17 temporal lobes data without artifact (Integral value 130.6, centroid value 62.7s). Integral analysis used the last $10 \mathrm{~s}$ of the pre-task, $60 \mathrm{~s}$ task, and first $55 \mathrm{~s}$ of the post-task $=125 \mathrm{~s}$ in total. These data were collected when she was in a calm, not manic, mood. Red line: oxyhemoglobin concentration, Blue line: deoxyhemoglobin concentration. 
A 27-year-old female presented at our out-patient service with binge eating as a main symptom. The first onset of binge eating appeared when she was a high school student. Her mood fluctuated between depressive to irritable (slight manic). She managed to address her binge eating with the help of a counselor; however, eating conduct was not controlled, so she was referred to our university hospital. She had bulimia nervosa as a coexisting illness. There was no reported family history of any specific diseases. Delusions or hallucinations were not observed, and no medication had been prescribed. The GAF score was 50. Other diseases that can cause mood change were ruled out by blood test including thyroidal, adrenal and iron function. First she was diagnosed with an eating disorder. Next, after a more detailed interview, she was diagnosed with bipolar disorder, type II, according to DSM-5. The mean centroid value of 93.2 in the frontal lobes indicated that this was non-major depressive disorder (Figure 3).

\section{Discussion}

In this clinical study, we evaluated the diagnostic accuracy between results from the subjective symptom (BDI-II) questionnaire and the BDI-II questionnaire with NIRS. There is a possibility of slight improvement in depression diagnosis accuracy when using NIRS compared with subjective symptoms alone, especially in the BDI-II low group. Using both the BDI-II and NIRS can evaluate patients subjectively and objectively. Further, both diagnoses are non-invasive. If diagnostic accuracy increases by the combining the questionnaire and NIRS, using NIRS in clinical practice will be useful. A previous study has reported a relationship between Hamilton's rating score for depression and NIRS [23]. Although diagnosis based on experienced experts is preferable, administration of these tests is complex for non-experts, and the diagnosis per se is not robust in terms of reproducibility. The use of objective tools, such as NIRS, could increase both diagnostic accuracy and objectivity. The diagnostic accuracy was higher in lower BDI-II score group when compared with the higher group. This may indicate that the use of NIRS for the diagnosis of depression would be more effective in primary care.

In addition, we compared with NIRS results of atypical depression with melancholic depression and bipolar disorder. Previous studies $[19,21,26]$ have argued that atypical depression may be included in bipolar disorder II or mild bipolar disorder. The NIRS image of atypical depression was similar to major depressive disorder when compared with bipolar disorder according to low centroid value. Therefore, we consider atypical depression is a subtype of major depressive disorder by the classification of DSM5 [17]. NIRS analysis of the temporal lobes is not common for the diagnosis of depression; however, a previous study reported that NIRS differentiated melancholic depression and non-melancholic depression in temporal lobes [30]. This indicates that NIRS imaging in the temporal lobes should be considered for future research. Previous studies [31-33] have reported that individuals diagnosed with bipolar disorder, who have a family history of this disease, tend to have more severe symptoms, such as early onset, and a high prevalence of suicide and psychosis.

One previous study has suggested that these patients have different brain structures when compared with patients with no family history of disease [34]. The individual diagnosed with bipolar disorder in this study does not have a family history; therefore, the results are not comparable. However, variations in family history may affect differences in NIRS images between bipolar disorders. Atypical depression is associated with metabolic diseases, such as diabetes and metabolic syndrome [14,16,35]. Therefore, atypical depression has both body and mind symptoms, and clinicians have to judge patients psychosomatically. This article will help psychosomatic medicine practitioners when debating similarities and differences between depression and psychosomatic illness, such as atypical depression. There are several limitations of this study. First, we performed a secondary analysis on our previous study data and compared the NIRS results from cases of atypical depression, melancholic depression, and bipolar disorder. The generalizability of these cases is not robust. Therefore, additional data and quantifiable analysis are necessary to consider impacts of confounding factors, such as medication use, as carefully as possible.

Nonetheless, to the best of our knowledge, this is the first study to compare the NIRS images of atypical and other types of depression. Second, we used the BDI-II for questionnaire in the study: however, there is a more appropriate questionnaire for evaluating major depressive disorder based on the DSM-5, such as the Patient Health Questionnaire (PHQ). To confirm our results, a more robust study using the PHQ is required. Third, we presented NIRS data for three cases of melancholic depression, atypical depression, and bipolar disorder respectively; however only three cases cannot confirm real differences, and a larger study is required. Nonetheless, this study is the first step to understanding NIRS data comparing subtypes of depression. Finally, BDI data for each case were not recorded on the NIRS measurement day. In the bipolar case, depression severity data would strengthen robustness of the results. Major depressive disorder will be ranked as the leading contributor to the global burden of disease in the near future [13]. All clinicians have to deal with depressive patients. A non-invasive objective assessment for depression may be necessary for every clinician $[3,36,37]$. To date, NIRS is too expensive and complex to manage; however, we believe that there is a possibility for a more reasonable tool. Our findings support the widespread clinical use of NIRS as an objective diagnostic tool for depression in the future.

\section{Conclusion}

In this paper, we addressed the possible role of NIRS as a diagnostic tool for depression and reported cases of NIRS diagnosis in atypical depression that related to the body and mind, in contrast to melancholic types of depression and bipolar disorders. 


\section{Author Contributions}

The first author, Takeaki Takeuchi, designed the study protocol and wrote the paper. The second author, Kazuaki Hashimoto, collected and analyzed the data, and discussed the interpretation of the data. The last author, Masahiro Hashizume designed the study and discussed the interpretation of the data.

\section{Funding}

This research received no external funding" or "This research was funded by Grant-in-Aid for Scientific Research (2016-2018) (No. 16K01799).

\section{Acknowledgment}

We would like to thank Editage (www.editage.jp) for English language editing.

\section{References}

1. Watanabe E, Yamashita Y, Maki A, Ito Y, Koizumi H (1996) Non-invasive functional mapping with multichannel near infra-red spectroscopic topography in humans. Neurosci. Lett 205(1): 41-44.

2. Schroeter ML, Bücheler MM, Müller K, Uludağ K, Obrig H, et al. (2004) Towards a standard analysis for functional near-infrared imaging. Neuroimage 21(1): 283-290.

3. Ferrari M, Quaresima VA (2012) A brief review on the history of human functional near-infrared spectroscopy (fNIRS) development and fields of application. Neuroimage 63(2): 921-935.

4. Scholkmann F, Kleiser S, Metz AJ, Zimmermann R, Mata Pavia J, et al. (2014) A review on continuous wave functional near-infrared spectroscopy and imaging instrumentation and methodology. Neuroimage 85 Pt 1: 6-27.

5. Jöbsis FF (1977) Noninvasive, infrared monitoring of cerebral and myocardial oxygen sufficiency and circulatory parameters. Science 198(4323): 1264-1267.

6. Bale G, Elwell CE, Tachtsidis I (2016) From Jöbsis to the present day: a review of clinical near-infrared spectroscopy measurements of cerebral cytochrome-c-oxidase. J Biomed Opt 21(9): 091307.

7. Kato H, Izumiyama M, Koizumi H, Takahashi A, Itoyama Y (2002) Near-infrared spectroscopic topography as a tool to monitor motor reorganization after hemiparetic stroke: a comparison with functional MRI. Stroke 33(8): 2032-2036.

8. Hock C, Villringer K, Müller Spahn F, Wenzel R, Heekeren H (1997) Decrease in parietal cerebral hemoglobin oxygenation during performance of a verbal fluency task in patients with Alzheimer's disease monitored by means of near-infrared spectroscopy (NIRS) correlation with simultaneous rCBF-PET measurements. Brain Res 755(2): 293-303.

9. Schytz HW, Guo S, Jensen LT, Kamar M, Nini A, et al. (2012) A new technology for detecting cerebral blood flow: A comparative study of ultrasound tagged NIRS and 133Xe-SPECT. Neurocrit Care 17(1): 139145.

10. Kawano M, Kanazawa T, Kikuyama H, Tsutsumi A, Kinoshita S, et al. (2016) Correlation between frontal lobe oxy-hemoglobin and severity of depression assessed using near-infrared spectroscopy. J Affect Disord 205: 154-158.

11. World Health Organization (2017) “Depression: let's talk” says WHO, as depression tops list of causes of ill health.

12. Takeuchi T, Nakao M, Nomura K, Inoue M, Tsurugano S, et al. (2009) Association of the metabolic syndrome with depression and anxiety in
Japanese men: a 1-year cohort study. Diabetes. Metab Res Rev 25(8): 762-767.

13. Pan A, Keum N, Okereke OI, Sun Q Kivimaki M, et al. (2012) Bidirectional association between depression and metabolic syndrome: a systematic review and meta-analysis of epidemiological studies. Diabetes Care 35(5): 1171-1180.

14. (2013) Diagnostic and statistical manual of mental disorders, 5th ed.: DSM-5. DC: American Psychiatric Association, Washington.

15. Hirschfeld RM (2014) Differential diagnosis of bipolar disorder and major depressive disorder. J Affect Disord 169(Suppl 1): S12-16.

16. Perugi G, Akiskal HS, Lattanzi L, Cecconi D, Mastrocinque C, et al. (1998) The high prevalence of "soft"bipolar (II) features in atypical depression. Compr Psychiatry 39(2): 63-71.

17. Benazzi F (1999) Prevalence of bipolar II disorder in atypical depression. Eur Arch Psychiatry Clin Neurosci 249(2): 62-65.

18. Perugi G, Fornaro M, Akiskal HS (2011) Are atypical depression, borderline personality disorder and bipolar II disorder overlapping manifestations of a common cyclothymic diathesis? World Psychiatry $10(1): 45-51$

19. Takeuchi T, Nakao M, Yano E (2006) Screening for major depression in the workplace: testing diagnostic accuracy of a two-item questionnaire used during mandatory testing. PRIMARY CARE COMMUN 11(1): 13-19.

20. Kanda Y (2013) Investigation of the freely available easy-to-use software 'EZR' for medical statistics. Bone Marrow Transplant 48(3): 452-458.

21. Sheehan DV, Lecrubier Y, Sheehan KH, Amorim P, Janavs J, et al. (1998) The Mini-International Neuropsychiatric Interview (M.I.N.I.): the development and validation of a structured diagnostic psychiatric interview for DSM-IV and ICD-10. J Clin Psychiatry 59 Suppl 10: 22-33.

22. Kawano M, Kanazawa T, Kikuyama H, Tsutsumi A, Kinoshita S, et al. (2016) Correlation between frontal lobe oxy-hemoglobin and severity of depression assessed using near-infrared spectroscopy. J Affect Disord 205: $154-158$

23. Noda T, Yoshida S, Matsuda T, Okamoto N, Sakamoto, et al. (2012) Frontal and right temporal activations correlate negatively with depression severity during verbal fluency task: a multi-channel near-infrared spectroscopy study. J Psychiatr Res 46(7): 905-912.

24. Pendleton MG, Heaton RK, Lehman RA, Hulihan D (1982) Diagnostic utility of the Thurstone Word Fluency Test in neuropsychological evaluations. J Clin Neuropsychol 4(4): 307-317.

25. Pu S, Nakagome K, Yamada T, Yokoyama K, Matsumura H, et al. (2012) The relationship between the prefrontal activation during a verbal fluency task and stress-coping style in major depressive disorder: a near-infrared spectroscopy study. J Psychiatr Res 46(11): 1427-1434.

26. Takizawa R, Fukuda M, Kawasaki S, Kasai K, Mimura M, et al. (2014) Neuroimaging-aided differential diagnosis of the depressive state. Neuroimage 85 Pt 1: 498-507.

27. Zhang H, Dong W, Dang W, Quan W, Tian J, et al. (2015) Near-infrared spectroscopy for examination of prefrontal activation during cognitive tasks in patients with major depressive disorder: a meta-analysis of observational studies. Psychiatry Clin Neurosci 69(1): 22-33.

28. Herrmann MJ, Ehlis AC, Fallgatter AJ (2004) Bilaterally reduced frontal activation during a verbal fluency task in depressed patients as measured by near-infrared spectroscopy. J Neuropsychiatry Clin Neurosci 16(2): 170-175.

29. Schecklmann M, Dresler T, Beck S, Jay JT, Febres R, et al. (2011) Reduced prefrontal oxygenation during object and spatial visual working memory in unipolar and bipolar depression. Psychiatry Res 194(3): 378-384.

30. Tsujii N, Mikawa W, Akashi H, Tsujimoto E, Adachi T, et al. (2014) Right temporal activation differs between melancholia and nonmelancholic 
depression: a multichannel near-infrared spectroscopy study. J Psychiatr Res 55: 1-7.

31. Baldessarini RJ, Tondo L, Vazquez GH, Undurraga J, Bolzani L, et al. (2012) Age at onset versus family history and clinical outcomes in 1,665 international bipolar-I disorder patients. World Psychiatry 11(1): 40-46.

32. Lin PI, McInnis MG, Potash JB, Willour V, MacKinnon DF, et al. (2006) Clinical correlates and familial aggregation of age at onset in bipolar disorder. Am J Psychiatry 163(2): 240-246.

33. Potash JB, Chiu YF, MacKinnon DF, Miller EB, Simpson SG, et al. (2003) Familial aggregation of psychotic symptoms in a replication set of 69 bipolar disorder pedigrees. Am J Med Genet B Neuropsychiatr Genet 116B(1): 90-97.

\section{ISSN: 2574-1241}

DOI: 10.26717/BJSTR.2019.19.003303

Takeaki Takeuchi. Biomed J Sci \& Tech Res

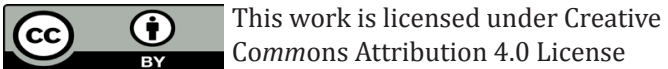

Submission Link: https://biomedres.us/submit-manuscript.php
34. Sharma V, Menon R, Carr TJ, Densmore M, Mazmanian D, et al. (2003) An MRI study of subgenual prefrontal cortex in patients with familial and non-familial bipolar I disorder. J Affect Disord 77(2): 167-171.

35. Chireh B, Li M, DArcy C (2019) Diabetes increases the risk of depression: A systematic review, meta- analysis and estimates of population attributable fractions based on prospective studies. Prev Med Rep 14: 100822.

36. Strangman G, Boas DA, Sutton JP (2002) Non-invasive neuroimaging using near-infrared light. Biol Psychiatry 52(7): 679-693.

37. Obrig H, Villringer A (2003) Beyond the visible--imaging the human brain with light. J Cereb Blood Flow Metab 23(1): 1-18.

$\begin{array}{ll}\text { BIOMEDICAL } & \text { Assets of Publishing with us } \\ \text { RESEARCHES } & \text { - Global archiving of articles } \\ & \text { - Immediate, unrestricted online access } \\ & \text { - Rigorous Peer Review Process } \\ \end{array}$

